\title{
New roles for "old" microRNAs in nervous system function and disease
}

\author{
Marion Hartl ${ }^{1,2}$ * and Ilona C. Grunwald Kadow ${ }^{2}$. \\ ${ }^{1}$ MRC Clinical Science Center, Hammersmith Hospital Campus, London, UK \\ ${ }^{2}$ Max-Planck Institute of Neurobiology, Martinsried, Germany
}

\section{Edited by:}

Hermona Soreq, The Hebrew

University of Jerusalem, Israel

Reviewed by:

Enrico Tongiorgi, University of Trieste, Italy

Marcos R. Costa, Federal University of Rio Grande do Norte, Brazil

\section{${ }^{*}$ Correspondence:}

Ilona C. Grunwald Kadow, Max-Planck Institute of Neurobiology, Am

Klopferspitz 18, 82152 Martinsried,

Germany

e-mail: ikadow@neuro.mpg.de

Marion Hartl, MRC Clinical Science

Center, Hammersmith Hospital

Campus, Du Cane Road, London

W12 ONN, UK

e-mail:m.hart|@csc.mrc.ac.uk
Since their discovery, microRNAs became prominent candidates providing missing links on how to explain the developmental and phenotypical variation within one species or among different species. In addition, microRNAs were implicated in diseases such as neurodegeneration and cancer. More recently, the regulation of animal behavior was shown to be influenced by microRNAs. In spite of their numerous functions, only a few microRNAs were discovered by using classic genetic approaches. Due to the very mild or redundant phenotypes of most microRNAs or their genomic location within introns of other genes many regulatory microRNAs were missed. In this review, we focus on three microRNAs first identified in a forward genetic screen in invertebrates for their essential function in animal development, namely bantam, let-7, and miR-279. All three are essential for survival, are not located in introns of other genes, and are highly conserved among species. We highlight their important functions in the nervous system and discuss their emerging roles, especially during nervous system disease and behavior.

\section{Keywords: let-7, bantam, miR-279, nervous system, development, behavior, regeneration, degeneration}

\section{INTRODUCTION}

The discovery of microRNAs was a big step towards the understanding of post-transcriptional regulation of gene expression. Several hundreds of microRNAs capable of interacting with a plethora of target mRNAs were discovered in model organisms. While sequence based prediction tools of microRNA-target interaction evolved quickly, phenotypical analysis of microRNA function lagged behind, in part due to the lack of clear phenotypes in mutants of single microRNAs. In addition, phenotypes were expressed only under certain environmental and experimental conditions suggesting that microRNAs act predominately by fine-tuning gene expression. As a consequence, microRNAs were rarely discovered in genetic screens. However, three microRNAs were found in genetic forward screens in invertebrates. All three are essential for survival, are not located in introns of other genes, and are highly conserved among species.

\section{INITIAL DISCOVERY OF let-7, bantam, AND miR-279}

Let-7 was the first microRNA described in C. elegans. It is conserved across different species and found in all common model organisms. In C. elegans let-7 participates in the so-called heterochronic pathway, which regulates the transition between different developmental stages in the worm by timing the division and differentiation of stem cells. Let-7 is upregulated in the last larval stage (L4), and by downregulating lin- $41 \mathrm{mRNA}$, allows the animal to fully mature. Weak mutant alleles of let-7 lead to a reiteration of larval patterns of cell division, and the animal fails to differentiate. Strong alleles of let-7 mutants cause a severe phenotype of a blasting vulva (Reinhart et al., 2000). The second example, bantam, has no mammalian homolog and has been intensively studied in
Drosophila. Mutations of this microRNA affect the proliferation of the wing disk and lead to failure in the G1-S transition of wing disk cells (Brennecke et al., 2003; Herranz et al., 2008). The third microRNA, miR-279, is highly conserved in insects. The first phenotype described was found in the olfactory system of Drosophila. In this system, miR-279 regulates the differentiation of a subclass of olfactory receptor neurons by downregulating two transcription factors, Nerfin-1 and Escargot (Cayirlioglu et al., 2008; Hartl et al., 2011).

Here, we review recent advances in the understanding of the function of let-7, bantam, and miR-279 in neural development, regeneration and degeneration, and behavior.

\section{Let-7 REGULATES CELLULAR DIFFERENTIATION OF THE NMJ AND OTHER BRAIN STRUCTURES}

In silico analysis predicted a strong interaction of let-7 with the transcription factor Abrupt. Recent studies verified this microRNA-target relationship in the neuromuscular junction (NMJ) and the mushroom body (MB) in Drosophila. In both tissues, the microRNA controls the developmental transition to an adult shape. When missing, the NMJ retains a juvenile shape and is not able to fully differentiate (Sokol et al., 2008). Although no anatomical phenotype was detected, let-7 mutant flies show defects in locomotion, flight, and also fertility (Sokol et al., 2008). The authors could show that the phenotype is accompanied by increased levels of the broad-complex, tramtrack, and bric-abrac (BTB) transcription factor Abrupt in let-7 mutants (Caygill and Johnston, 2008) corroborating a previous finding that Abrupt ensures the remodeling of the larval NMJ to achieve its adult shape and function (Hu et al., 1995). 
Two other recent studies in Drosophila show that the let-7 complex (let-7-C) is a key regulator of the development of the $\mathrm{MB}$ (Kucherenko et al., 2012; Wu et al., 2012). Let-7-C gives rise to three different microRNAs, namely (let-7, miR-100, and miR-125), which can act individually but also synergistically on mRNA regulation. The Drosophila MB is a complex brain structure essential for olfactory learning and memory as well as context-dependent innate behavior (Heisenberg, 2003; Fiala, 2008; Bracker et al., 2013). It is comprised of Kenyon cells (KCs) that can be further classified into four different subtypes. During development, they are derived from multi-potent progenitor cells and are born in a fixed order $\left(\gamma \rightarrow \alpha^{\prime} / \beta^{\prime} \rightarrow\right.$ pioneer $\alpha / \beta \rightarrow \alpha / \beta$; Zhu et al., $2003,2006)$. Two factors guiding the precise timing of MB neuron subtypes are the transcription factors, "Chronologically inappropriate morphogenesis" (Chinmo) and Abrupt (Zhu et al., 2006; Kucherenko et al., 2012). Chinmo affects the differentiation of $\mathrm{MB}$ subtypes in a concentration dependent manner. While high levels of Chinmo in post-mitotic neurons specify $\gamma$ and $\alpha^{\prime} / \beta^{\prime}$, low levels of Chinmo drive the differentiation of late-born MB neurons (pioneer $\alpha / \beta, \alpha / \beta$; Zhu et al., 2006). In order to generate different levels of Chinmo throughout $\mathrm{MB}$ development, let-7 and miR-125 co-transcribed from the let-7-C locus, contribute to the progressive downregulation of chinmo in vivo. Let-7 and miR125 regulate chinmo expression directly via binding sites in the $3^{\prime} \mathrm{UTR}$ of the transcription factor. The third microRNA of the let7-C, mir-100, seems not to be involved in the post-transcriptional regulation (Wu et al., 2012). All let-7-C microRNAs are strongly upregulated in the transition from the late pupal to early adult stage. In vivo, precocious expression of let-7 and miR-125 in larval stage 1 leads to a sharp decrease of Chinmo levels already in larval stage 3 . As a consequence, the adult $\mathrm{MB}$ shows strong morphological defects and mis-differentiation of its cell types. The second study revealed that let-7-C also influences the timing of $\alpha^{\prime} / \beta^{\prime}$ to $\alpha / \beta$ transition via the Chinmo related BTB transcription factor Abrupt (Kucherenko et al., 2012). The differentiation of the late born $\alpha / \beta$ neurons depends on the expression of let-7C. By contrast, Abrupt is essential to establish the identity of $\alpha^{\prime} / \beta^{\prime}$ neurons. Thus, let-7- $C$ mediated downregulation of Abrupt regulates the transition between different subsets of $\mathrm{MB}$ neurons. Notably, the expression of let-7-C appears to be dependent on Ecdysone signaling, a key regulator for morphological transitions during insect development (Robbins et al., 1968). While both studies describe effects on $\mathrm{MB}$ morphology, the effect of the let-7-C mutation on MB morphology differs in the two studies. Wu et al. only find delays in the transition towards different MB subtypes. By contrast, Kucherenko et al. detect a significant reduction of $\alpha / \beta$ lobe volume. Reasons for these phenotypical differences are not known, but may have to do with the role of microRNAs as buffers rather than instructors of gene expression. Therefore different experimental conditions such as nutrition and temperature might influence the severity of the phenotype. Nevertheless, the results of the two publications show that let-7-C is used to sharpen the expression of two potent transcription factors in order to produce different neuronal subtypes. While Chinmo has a broader effect on $\mathrm{MB}$ development and seems to affect the generation of all $\mathrm{MB}$ subtypes, Abrupt only affects the transition of the late born neurons.

\section{MECHANISMS OF let-7 REGULATION}

In order to precisely time the expression of potent transcription factors during development, microRNA expression needs to be tightly regulated in expression. To ensure precise timing of activity for instance during neuronal differentiation, let-7 interacts with one of its classical targets in an autoregulatory cycle. In embryonic stem (ES) and embryonic carcinoma (EC) cells, the pluripotency factor Lin-28 binds pre-let-7 and inhibits the last step during let-7 processing and thereby prevents the formation of a mature microRNA (Rybak et al., 2008). In neural stem cells, lin-28 is repressed by let-7 and $m i R-125$. This leads to a neural stem cell commitment towards differentiated neurons (Rybak et al., 2008).

The transcription factor SRY (sex-determining region)-box 2 (SOX2) directly binds the Lin-28 promotor and regulates its expression (Cimadamore et al., 2013). Expression and activation of Lin-28 inhibits let-7 and leads to the maturation of the NPCs, which are derived from human ES cells (Cimadamore et al., 2013). In this context, loss of SOX2 as well as overexpression of let-7 (specifically of let-7i) led to the inhibition of neuronal differentiation (Cimadamore et al., 2013).

\section{Bantam DETERMINES CELLULAR GROWTH IN THE NERVOUS SYSTEM}

During development, two processes must be coordinated: first, cells differentiate to obtain their cellular fate and function, and second, the cell number is multiplied forming the basis of growth and proliferation. Recent studies implicate the microRNA bantam in glia proliferation in the Drosophila brain and optic lobe. Drosophila larvae undergo an extreme growth in the third instar stage. Epidermal growth is often linked to the well-studied Hippo pathway. In a recent study, Reddy and Irvine (2011) show an involvement of the pathway in non-epithelial glia cells for the first time. In the Hippo pathway, Merlin acts as the upstream regulator of the core kinase cascade in the Hippo pathway. Merlin depletion or expression of an activated form of Yorkie leads to glia overgrowth in the optic lobe and the brain of Drosophila. Similar to the wing disk, Merlin activates the expression of Yorkie and in turn, Yorkie was found to activate the expression of bantam (Nolo et al., 2006; Thompson and Cohen, 2006). As a consequence of bantam, expression levels of Myc are increased, probably as an indirect effect due to suppression of the ubiquitin ligase Mei-P26 (Herranz et al., 2010). Interestingly, neurons are insensitive to increased levels of bantam and hence, glia cells are affected exclusively.

Another study describes a role for bantam during the development of another neural structure, the optic lobe. Bantam is highly expressed in mitotically active cells in the developing optic lobe. Depletion of bantam levels in third instar Drosophila larvae leads to smaller optic lobes, whereas overexpression of bantam results in an increased volume of optic lobes. The effect of bantam is not due to a mis-differentiation of glia cells since even in the full mutant larvae glia cells are present. In this context bantam seems to influence the number of glia cells. The authors conclude that bantam maintains the pool of stem cells during development and thus influences the proliferation of the cells. In the same study, the T-box transcription factor Omb was identified as downstream target of bantam that can also partially rescue the gain-of-function phenotype of the microRNA. The molecular mechanism of how 
bantam controls the cell cycle remains to be investigated ( $\mathrm{Li}$ and Padgett, 2012).

\section{miR-279 AS A MOLECULAR SWITCH OF $\mathrm{CO}_{2}$ NEURON LOCATION}

miR-279 is highly conserved in all insect species, and was first identified in a forward genetic screen for axon guidance and synapse formation factors using the olfactory system of Drosophila as a model system (Cayirlioglu et al., 2008). The olfactory system in Drosophila consists of two appendages, the antenna and the maxillary palp, which house different sets of olfactory sensory neurons (OSNs). During development each subset of OSNs sends their axons to a defined area, a glomerulus, in the antennal lobe in the brain to generate a spatial representation of odors. In the olfactory system, miR-279 regulates development and axon targeting of a specific class of sensory neurons. Flies mutant for $m i R-279$ develop extra $\mathrm{CO}_{2}$ sensory neurons on the second olfactory appendage of insects, the maxillary palp, in addition to $\mathrm{CO}_{2}$ neurons found on control antenna (Jones et al., 2007; Kwon et al., 2007). These ectopic $\mathrm{CO}_{2}$ neurons innervate a glomerulus in the antennal lobe associated with the detection of food cues and not with the detection of $\mathrm{CO}_{2}$ (Cayirlioglu et al., 2008) The innervation pattern of the ectopic $\mathrm{CO}_{2}$ neurons highly resembles the location and central brain innervation of mosquito $\mathrm{CO}_{2}$ neurons (Ghaninia et al., 2007; Lu et al., 2007). Therefore, $m i R-279$ was proposed as a molecular switch in the divergence of mosquitoes and flies for the trait of $\mathrm{CO}_{2}$ detection (Cayirlioglu et al., 2008; Jones, 2008). Since flies are highly repelled by $\mathrm{CO}_{2}$ and mosquitoes strongly attracted to it the study provides a starting point to explore how molecular changes in regulation shape neural circuits and thereby the behavioral output (Benton, 2008; Jones, 2008). Similar to let-7, miR-279 appears to regulate neuronal commitment and differentiation of progenitor cells (Cayirlioglu et al., 2008; Hartl et al., 2011; Table 1). On the mechanistic level, $m i R-279$ expression is regulated by the pan-neuronal transcription factor Prospero
(Hartl et al., 2011), an important player in stem cell progression and sensory neuron development (Doe et al., 1991; Choksi et al., 2006). Two transcription factors both of which are also direct targets of Prospero, the snail transcription factor Escargot and the zinc-finger transcription factor Nerfin-1 were identified as essential and sufficient targets of $m i R-279$ : gain-of-function of both targets together induced the formation and mistargeting of ectopic $\mathrm{CO}_{2}$ neurons efficiently (Hartl et al., 2011). Thus, the pan-neural factor Prospero refines its own activity by inducing a microRNA to regulate the expression of its own downstream target genes. Given Prospero's role during stem cell development in both flies and mice a use of the same or similar network is conceivable also in the control of tumor formation in the brain or the lymphatic system (Petrova et al., 2002; Galeeva et al., 2007).

\section{NEURONAL REGENERATION}

While aging, the nervous system progressively loses the ability to rapidly regenerate new cells. The decline in regeneration during aging is a conserved phenomenon. In C. elegans, the anterior ventral microtubule (AVM) axon is used to study the effect of single molecules on the regeneration of neurons. The regeneration decline in C. elegans occurs usually in the larval stage 3 (L3) prior to the transition to young adults. Worms mutant for microRNA biosynthesis factors Dicer-1 or Argonaute Alg-1, however, continue to regenerate the axons of AVM which in turn extend much longer as compared to wildtype controls (Zou et al., 2013). In this context, the microRNA involved and responsible for increased axonal length and regeneration is let-7 (Figure 1). Mutants of let7 exhibited the same phenotype in AVM neurons as alg-1 mutants. The study showed that in order to stop AVM axons from extending, Lin-41 is strongly repressed by let-7 in late adult stages. Mutants of lin-41 show a decreased regeneration of the axons. Interestingly, Lin-41 co-immunoprecipitates with Alg-1, which constitutes a key factor for let-7 biogenesis. The experiments suggest that in

Table 1 | Conserved functions of let-7, bantam, and miR-279 in the nervous system.

\begin{tabular}{|c|c|c|c|c|}
\hline & microRNA & Model organism & Function & Citation \\
\hline \multirow[t]{6}{*}{ Development } & let-7 & Drosophila & Maturation of neuromuscular junction (NMJ) & Caygill and Johnston (2008), Sokol etal. (2008) \\
\hline & & & Mushroom body (MB) differentiation & Kucherenko etal. (2012), Wu etal. (2012) \\
\hline & & $\begin{array}{l}\text { Human neural } \\
\text { precursor cells } \\
\text { embryonic stem cells }\end{array}$ & Pluripotency & Rybak etal. (2008), Cimadamore etal. (2013) \\
\hline & bantam & Drosophila & Glia cell growth in the brain and optic lobe & Reddy and Irvine (2011) \\
\hline & & & $\begin{array}{l}\text { Differentiation and number of glia cells in the } \\
\text { optic lobe }\end{array}$ & Li and Padgett (2012) \\
\hline & $\operatorname{miR}-279$ & Drosophila & $\mathrm{CO}_{2}$ neuron development & Cayirlioglu et al. (2008), Hartl et al. (2011) \\
\hline \multirow[t]{3}{*}{ Regeneration } & let-7 & C. elegans & AVM neuron axon regeneration & Zou et al. (2013) \\
\hline & & Zebrafish & De-differentiation of Mueller glia cells & Ramachandran et al. (2010) \\
\hline & bantam & Drosophila & Dendritic aborisation (da) neuron regeneration & Song etal. (2012) \\
\hline Degeneration & let-7 & Mouse & Loss of cortical neurons through extracellular let-7 & Lehmann et al. (2012) \\
\hline Behavior & $\operatorname{miR}-279$ & Drosophila & Regulation of circadian rhythm & Luo and Sehgal (2012) \\
\hline
\end{tabular}




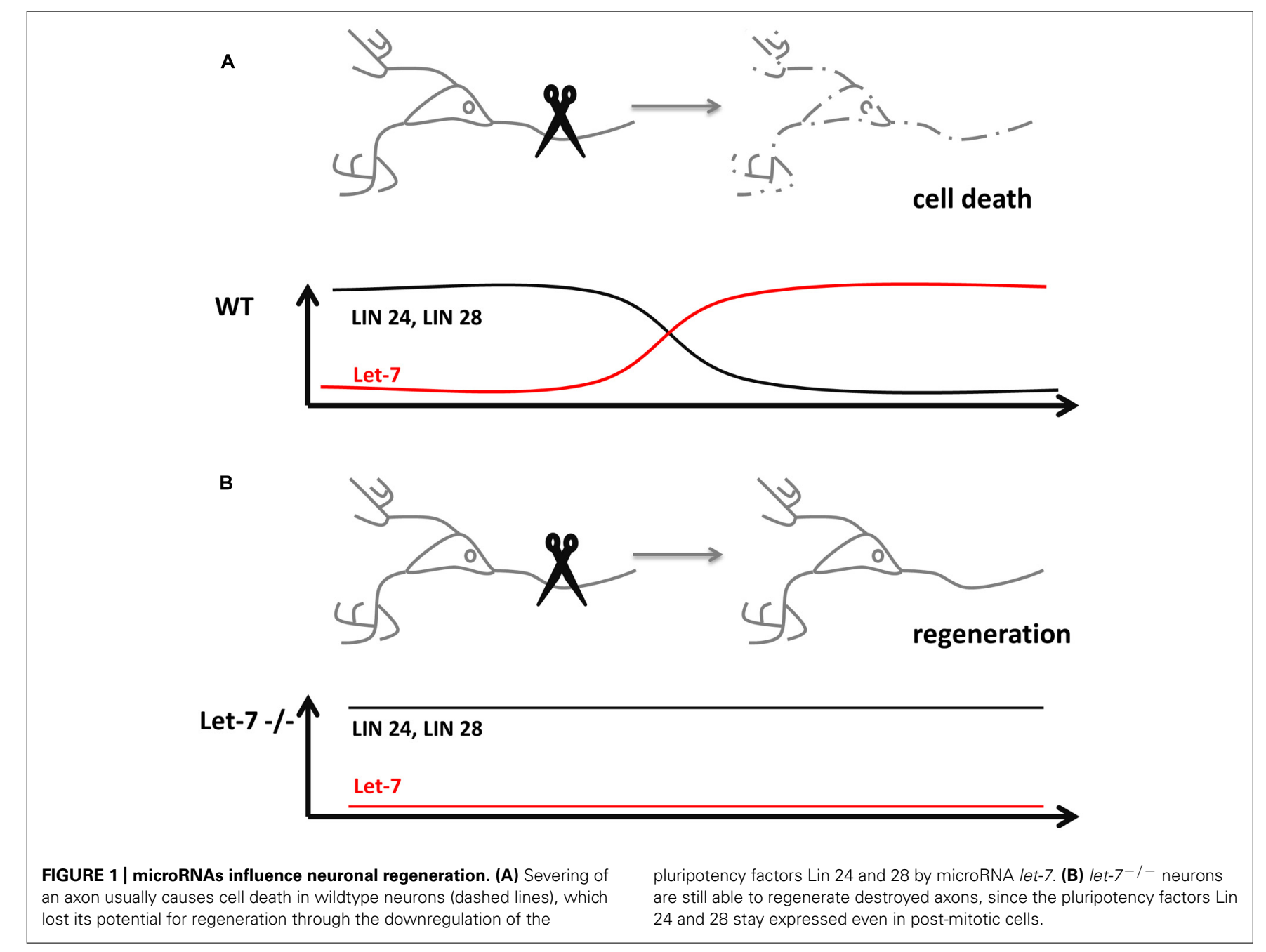

early stages of development a Lin-41/Alg-1 complex is formed and represses the synthesis of let-7 permitting axonal regeneration and extension. In late larval up to the young adult stages, this suppression is removed and let-7 is processed to the mature microRNA, which effectively downregulates lin-41. In turn, the AVM axons are no longer able to regenerate and stop to grow. Therefore, the molecular mechanism underlying the regeneration decline of AVM axons exemplifies, how a regulatory circuit is reused in post-mitotic cells.

Another example of how a pluripotency factor is used to trigger regeneration in post-mitotic cells was studied in the fish retina. The retina of fish has the remarkable potential to fully regenerate after injury. In order to recover, Müller glia cells de-differentiate and form new progenitors. The regeneration potential is based on the high expression of the pluripotency factor Lin-28 in dedifferentiated Müller glia cells, a feature shared with ES cells (Rybak et al., 2008; Table 1). Since Lin-28 is also a known target of let-7, the fish retina exemplifies the autoregulatory mechanism of the microRNA and its target in a regeneration inducing process (Ramachandran et al., 2010).

In another system, the dendritic arborisation (da) neurons found in the body wall of Drosophila larvae, microRNA bantam was found to be involved in the process of balancing the growth of dendrites and the underlying epithelium as the target area of the neurons. During a process called scaling, the microRNA functions as a signal to synchronize the neuronal growth with the epithelium. When bantam is missing, the dendrites overshoot and fail to cover the appropriate space. Interestingly, during this process, bantam is not expressed in the neurons but in the epithelial target cell and acts as a signal to downregulate Akt signaling in the neurons. How the microRNA signal is transferred is not yet found and opens up a new field of study dealing with microRNA signal transduction via direct microRNA transport between neighboring cells (Parrish et al., 2009). In a new study, the axons and dendrites of one class of da neurons are established as a model for neuronal regeneration (Song et al., 2012). Similar to the mammalian system, peripheral sensory neurons retain the potential to regenerate after injury, whereas the processes of central neurons fail to regenerate after injury. Studies how to overcome this lack of regeneration were mostly performed in the mammalian system. However, this study shows in detail that the da neurons in the Drosophila body wall and the ventral nerve cord can serve as a model to identify the molecular players involved in regeneration. 
Not only in the axons, also da neuron dendrites in bantam mutants regenerate. The effect could also be mimicked through depletion of PTEN or gain-of-function of Akt in the neurons. Because Akt signaling is involved in scaling as well as in regeneration of the da neurons, regulatory cycles important during are likely reused during regeneration.

\section{Let-7 IS INVOLVED IN THE DEGENERATION OF NEURONS}

An unexpected role of let-7 was revealed in a study on signaling mechanisms leading to neuronal degeneration (Lehmann et al., 2012). Upon a neuronal damage, e.g., during the course of Alzheimer's disease, the immune system multiplies the degradation process through a so far unidentified mechanism. In a recent study, the RNA sensing receptor Toll-like 7 (TLR 7) in cortical neurons of mice was shown to bind extracellular enriched let-7 released by degenerating neurons (Figure 2). Subsequently, the TLR 7 expressing cell undergoes apoptosis. Mice injected with let$7 b$ into the spinal canal lose $18 \%$ of neurons after 3 days in the cortical area where TLR 7 is endogenously expressed. A comparable loss of neurons occurred also in the striatal area of the mouse brain. After 2 weeks, the effect further increased up to a loss of $30 \%$ of neurons. In mice lacking the TLR 7 receptor, no neuronal loss was detectable. Injection of let-7b was in turn also sufficient to activate downstream TLR 7 signaling which was shown by the increased phosphorylation state of IRAK4 (Takeuchi and Akira, 2010).

Moreover, an increase of extracellular let-7b was also measured in the corticospinal fluid (CSF) of Alzheimer patients (Lehmann et al., 2012). This observation implies a conserved function of the microRNA as signaling molecule in neurodegenerative diseases. Whether depletion of let-7b could decrease the extent of neurodegeneration in Alzheimer disease patients appears to be an interesting hypothesis to be tested in future studies.

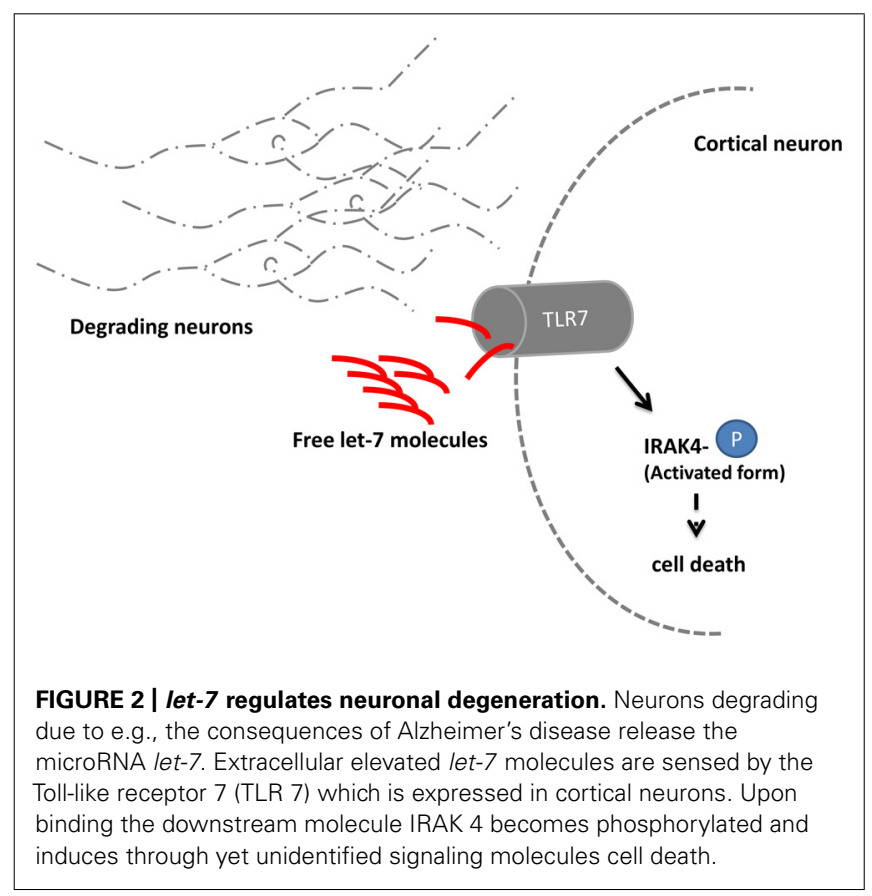

\section{microRNAs CONTROL BEHAVIOR}

Recent work implicated microRNA function also in the behavior of adult animals. Using an overexpression screen, Luo and Sehgal (2012) demonstrated that miR-279 controls the circadian rhythm of flies (Figure 3). Enhanced levels of miR-279 were found to disrupt the rest-activity cycles of Drosophila. Cycles in circadian rhythm are linked to the oscillations of Period in so-called pacemaker neurons (Ozkaya and Rosato, 2012). These oscillations are normal in miR-279 mutants suggesting that the microRNA is an effector of Period transmitting signals from pacemaker neurons. Interestingly, miR-279 targets the secreted morphogen unpaired (Upd) using a JAK/STAT-dependent interaction that was recently discovered in the oocyte (Yoon et al., 2011). The Drosophila ovaries contain migratory border cells and non-migratory follicle cells. A gradient of the secreted morphogen unpaired (Upd) is used to establish the cell fate of the two subtypes (Yoon et al., 2011). In order to activate JAK/STAT signaling, the cytokine Upd is secreted and binds STAT (signal transducer and activator of transcription). High levels of Upd specify migratory border cells (Silver and Montell, 2001; Ghiglione et al., 2002), whereas low or transient levels specify the non-migratory follicle cells (Starz-Gaiano et al., 2008). In the oocyte, $m i R-279$ was found to favor the cell fate of follicle cells through repression of STAT. In the adult fly, reducing the levels of Upd in miR-279 mutants rescues the circadian rhythm phenotype. Central pacemaker neurons target neurons positive for Upd and were proposed to be candidates for signal receivers of the central clock. This study shows for the first time, which signaling pathway transmits the PER protein oscillations of the circadian clock to downstream neurons. In addition, it represents one of the rare examples for a function of microRNAs in behavior without affecting the development of the underlying circuits.

\section{DISCUSSION}

Mutants of only a few microRNAs were found to be essential or to exhibit substantial phenotypes. Of the characterized microRNAs, most appear to fine-tune expression of target mRNAs. The microRNAs with a quantifiable and persistent phenotype are good candidates to study microRNA function and regulation in detail. Three microRNAs, let-7, bantam, and miR-279 were discovered in forward genetic screens, because their mutants showed substantial developmental phenotypes. Notably, these microRNAs are encoded by their own genetic loci, in contrast to microRNAs that are found in intronic regions of other genes. In this review, we focused on the roles of these well studied microRNAs in the nervous system. Besides multiple developmental effects of these microRNAs, more recent studies find them to be involved in regeneration, degeneration, and also behavior.

During development the discussed microRNAs target very often pluripotency factors to ensure the proper transition between differentiation and proliferation of different cell types. In mutants, cells fail to differentiate or properly grow. Interestingly, the same microRNA target relationships also affect regeneration events in post-mitotic neurons or glia cells. As exemplified in the fish retina and fly da neurons blocking the expression of let-7 or bantam enables the cell to increase the levels of pluripotency factors to allow cell growth and thereby regeneration of cells and tissues. 

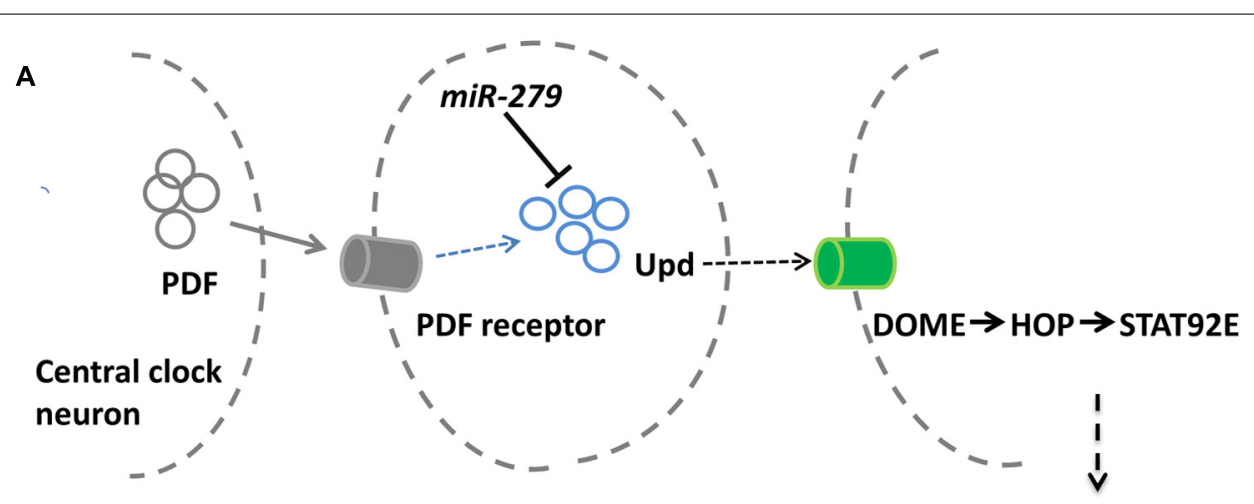

\section{Rest:Activity Cycle}

B

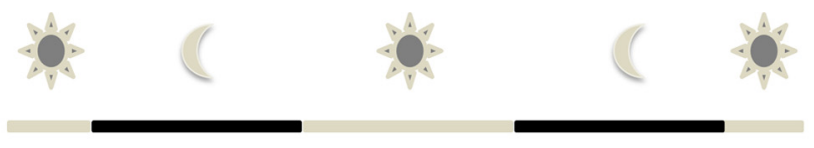

wildtype

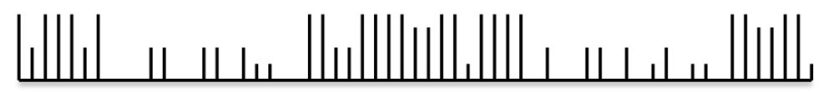

miR-279 mutant

FIGURE 3 | miR-279 regulates the circadian rhythm in Drosophila.

(A) Schematic showing the molecular mechanisms of central clock neurons to downstream neurons in order to regulate the rest/activity cycle. The central clock neurons release PDF which is sensed via the pdf receptor in target cells. Upon PDF receptor activation, the expression of the cytokine Unpaired is activated, but also repressed via the microRNA miR-279. The released cytokine is bound by the receptor DOME in central clock downstream neurons which eventually assure the transition between rest and activity in the circadian rhythm of the fly. (B) During the day a wildtype fly shows alternating patterns of activity and rest. During daytime the fly shows high activity which is indicated by the high bars in the activity plot. During the night the rest cycle starts, indicated by low bars. The microRNA-279 mutant flies do not show rest/activity cycles anymore and show a overall activity throughout day and night.
In contrast, let-7 uses a new and different mechanism in the context of neuronal degeneration. Here, an increase in let-7 levels in degenerating neurons induces cell death of cortical neurons. In this case, let-7 acts as an extracellular signaling molecule. Two very interesting questions arise from this finding: (i) how are microRNAs transported? and (ii) do other microRNAs work as signaling molecules? The type of microRNAs and the mechanisms of microRNA-mediated extracellular signaling might also be of interest in light of a potential use of microRNAs as therapeutic agents to prevent neuronal cell loss in neurodegenerative diseases such as Alzheimer's (Chen etal., 2012). We also discussed a study of a novel role of $m i R-279$ during the circadian rhythm regulation in Drosophila. This study provided an interesting example of the function of small regulatory RNAs in the adult animal behavior. The authors demonstrated that miR-279 helps transmitting the JAK/STAT pathway signal to neurons downstream of pace maker neurons without affecting their development. This study is remarkable, because it showed for the first time that oscillations of Period levels are transmitted to downstream neurons. Regarding the role of microRNAs, the work encourages a search for additional microRNAs involved in the regulation of behavior.

However, the cases of the three discussed microRNAs also imply that the approach of identifying microRNAs through a forward screen approach has weaknesses, because of the highly redundant function of the majority of these molecules. Through the development and further refinement of RNAseq methods, it is now possible to identify the set of microRNAs expressed in a specific tissue or even in a single cell. Alternatively to forward genetic screens, RNAseq mediated analysis of expression of all microRNAs in a given sample could be used to specifically manipulate a combination of microRNAs. These manipulations are being facilitated by the existence of new methods such as site-directed excision with Zinc finger nucleases (Zfn), TALENs (transcription activator-like effector nuclease), or CRISPR-Cas (clustered regulatory interspaced short palindromic repeat; Gaj et al., 2013). Using a modified reverse genetic approach the function of a specific microRNA or groups of microRNAs could be revealed.

Thanks to the work of many groups, we have gained a substantial understanding of microRNA function during development of the nervous system and beyond. The recent findings that 
microRNAs regulate aspects of adult animal biology such as neuronal degeneration and regeneration as well as behavior suggest that still much is to be discovered to complete our picture of microRNA-mediated gene regulation and signaling. New genetic and genomic approaches and constantly improved methods to monitor gene expression will pave the way to analyze the function not only of single essential microRNAs but also of groups of microRNAs during nervous system development and in the adult animal.

\section{REFERENCES}

Benton, R. (2008). Chemical sensing in Drosophila. Curr. Opin. Neurobiol 18, $357-$ 363. doi: 10.1016/j.conb.2008.08.012

Bracker, L. B., Siju, K. P., Varela, N., Aso, Y., Zhang, M., Hein, I., et al. (2013). Essential role of the mushroom body in context-dependent $\mathrm{CO}(2)$ avoidance in Drosophila. Curr. Biol. 23, 1228-1234. doi: 10.1016/j.cub.2013.05.029

Brennecke, J., Hipfner, D. R., Stark, A., Russell, R. B., and Cohen, S. M. (2003). bantam Encodes a developmentally regulated microRNA that controls cell proliferation and regulates the proapoptotic gene hid in Drosophila. Cell 113, 25-36. doi: 10.1016/S0092-8674(03)00231-9

Caygill, E. E., and Johnston, L. A. (2008). Temporal regulation of metamorphic processes in Drosophila by the let-7 and miR-125 heterochronic microRNAs. Curr. Biol. 18, 943-950. doi: 10.1016/j.cub.2008.06.020

Cayirlioglu, P., Kadow, I. G., Zhan, X., Okamura, K., Suh, G. S., Gunning, D., et al. (2008). Hybrid neurons in a microRNA mutant are putative evolutionary intermediates in insect CO2 sensory systems. Science 319, 1256-1260. doi: $10.1126 /$ science. 1149483

Chen, X., Liang, H., Zhang, J., Zen, K., and Zhang, C. Y. (2012). Secreted microRNAs: a new form of intercellular communication. Trends Cell Biol. 22, 125-132. doi: 10.1016/j.tcb.2011.12.001

Choksi, S. P., Southall, T. D., Bossing, T., Edoff, K., De Wit, E., Fischer, B. E., et al. (2006). Prospero acts as a binary switch between self-renewal and differentiation in Drosophila neural stem cells. Dev. Cell 11, 775-789. doi: 10.1016/j.devcel.2006.09.015

Cimadamore, F., Amador-Arjona, A., Chen, C., Huang, C. T., and Terskikh, A. V. (2013). SOX2-LIN28/let-7 pathway regulates proliferation and neurogenesis in neural precursors. Proc. Natl. Acad. Sci. U.S.A. 110, E3017-E3026. doi: $10.1073 /$ pnas. 1220176110

Doe, C. Q., Chu-Lagraff, Q., Wright, D. M., and Scott, M. P. (1991). The prospero gene specifies cell fates in the Drosophila central nervous system. Cell 65, 451-464. doi: 10.1016/0092-8674(91)90463-9

Fiala, A. (2008). Neuroethology: a neuronal self-defense mechanism in fly larvae. Curr. Biol. 18, R116-R117. doi: 10.1016/j.cub.2007.11.054

Gaj, T., Gersbach, C. A., and Barbas, C. F. III. (2013). ZFN, TALEN, and CRISPR/Casbased methods for genome engineering. Trends Biotechnol. 31, 397-405. doi: 10.1016/j.tibtech.2013.04.004

Galeeva, A., Treuter, E., Tomarev, S., and Pelto-Huikko, M. (2007). A prosperorelated homeobox gene Prox-1 is expressed during postnatal brain development as well as in the adult rodent brain. Neuroscience 146, 604-616. doi: 10.1016/j.neuroscience.2007.02.002

Ghaninia, M., Hansson, B. S., and Ignell, R. (2007). The antennal lobe of the African malaria mosquito, Anopheles gambiae - innervation and three-dimensional reconstruction. Arthropod. Struct. Dev. 36, 23-39. doi: 10.1016/j.asd.2006.06.004

Ghiglione, C., Devergne, O., Georgenthum, E., Carballes, F., Medioni, C., Cerezo, D., et al. (2002). The Drosophila cytokine receptor Domeless controls border cell migration and epithelial polarization during oogenesis. Development 129, 5437-5447. doi: 10.1242/dev.00116

Hartl, M., Loschek, L. F., Stephan, D., Siju, K. P., Knappmeyer, C., and Kadow, I. C. (2011). A new Prospero and microRNA-279 pathway restricts CO2 receptor neuron formation. J. Neurosci. 31, 15660-15673. doi: 10.1523/JNEUROSCI.259211.2011

Heisenberg, M. (2003). Mushroom body memoir: from maps to models. Nat. Rev. Neurosci. 4, 266-275. doi: 10.1038/nrn1074

Herranz, H., Hong, X., Perez, L., Ferreira, A., Olivieri, D., Cohen, S. M., et al. (2010). The miRNA machinery targets Mei-P26 and regulates Myc protein levels in the Drosophila wing. EMBO J. 29, 1688-1698. doi: 10.1038/emboj.2010.69
Herranz, H., Perez, L., Martin, F. A., and Milan, M. (2008). A wingless and notch double-repression mechanism regulates G1-S transition in the Drosophila wing. EMBO J. 27, 1633-1645. doi: 10.1038/emboj. 2008.84

Hu, S., Fambrough, D., Atashi, J. R., Goodman, C. S., and Crews, S. T. (1995). The Drosophila abrupt gene encodes a BTB-zinc finger regulatory protein that controls the specificity of neuromuscular connections. Genes Dev. 9, 2936-2948. doi: 10.1101/gad.9.23.2936

Jones, W. D. (2008). MicroRNA mutant turns back the evolutionary clock for fly olfaction. Bioessays 30, 621-623. doi: 10.1002/bies.20780

Jones, W. D., Cayirlioglu, P., Kadow, I. G., and Vosshall, L. B. (2007). Two chemosensory receptors together mediate carbon dioxide detection in Drosophila. Nature 445, 86-90. doi: 10.1038/nature05466

Kucherenko, M. M., Barth, J., Fiala, A., and Shcherbata, H. R. (2012). Steroid-induced microRNA let-7 acts as a spatio-temporal code for neuronal cell fate in the developing Drosophila brain. EMBO J. 31, 4511-4523. doi: 10.1038/emboj.2012.298

Kwon, J. Y., Dahanukar, A., Weiss, L. A., and Carlson, J. R. (2007). The molecular basis of CO2 reception in Drosophila. Proc. Natl. Acad. Sci. U.S.A. 104, 3574-3578. doi: 10.1073/pnas.0700079104

Lehmann, S. M., Kruger, C., Park, B., Derkow, K., Rosenberger, K., Baumgart, J., et al. (2012). An unconventional role for miRNA: let-7 activates Toll-like receptor 7 and causes neurodegeneration. Nat. Neurosci. 15, 827-835. doi: 10.1038/nn.3113

Li, Y., and Padgett, R. W. (2012). bantam is required for optic lobe development and glial cell proliferation. PLoS ONE 7:e32910. doi: 10.1371/journal.pone.0032910

Lu, T., Qiu, Y. T., Wang, G., Kwon, J. Y., Rutzler, M., Kwon, H. W., et al. (2007). Odor coding in the maxillary palp of the malaria vector mosquito Anopheles gambiae. Curr. Biol. 17, 1533-1544. doi: 10.1016/j.cub.2007.07.062

Luo, W., and Sehgal, A. (2012). Regulation of circadian behavioral output via a microRNA-JAK/STAT circuit. Cell 148, 765-779. doi: 10.1016/j.cell.2011.12.024

Nolo, R., Morrison, C. M., Tao, C., Zhang, X., and Halder, G. (2006). The bantam microRNA is a target of the hippo tumor-suppressor pathway. Curr. Biol. 16, 1895-1904. doi: 10.1016/j.cub.2006.08.057

Ozkaya, O., and Rosato, E. (2012). The circadian clock of the fly: a neurogenetics journey through time. Adv. Genet. 77, 79-123. doi: 10.1016/B978-0-12-3876874.00004-0

Parrish, J. Z., Xu, P., Kim, C. C., Jan, L. Y., and Jan, Y. N. (2009). The microRNA bantam functions in epithelial cells to regulate scaling growth of dendrite arbors in Drosophila sensory neurons. Neuron 63, 788-802. doi: 10.1016/j.neuron.2009.08.006

Petrova, T. V., Makinen, T., Makela, T. P., Saarela, J., Virtanen, I., Ferrell, R. E., et al. (2002). Lymphatic endothelial reprogramming of vascular endothelial cells by the Prox-1 homeobox transcription factor. EMBO J. 21, 4593-4599. doi: 10.1093/emboj/cdf470

Ramachandran, R., Fausett, B. V., and Goldman, D. (2010). Asclla regulates Muller glia dedifferentiation and retinal regeneration through a Lin-28-dependent, let-7 microRNA signalling pathway. Nat. Cell Biol. 12, 1101-1107. doi: $10.1038 / \mathrm{ncb} 2115$

Reddy, B. V., and Irvine, K. D. (2011). Regulation of Drosophila glial cell proliferation by Merlin-Hippo signaling. Development 138, 5201-5212. doi: 10.1242/dev.069385

Reinhart, B. J., Slack, F. J., Basson, M., Pasquinelli, A. E., Bettinger, J. C., Rougvie, A. E., et al. (2000). The 21-nucleotide let-7 RNA regulates developmental timing in Caenorhabditis elegans. Nature 403, 901-906. doi: 10.1038/35002607

Robbins, W. E., Kaplanis, K. N., Thompson, M. J., Shortino, T. J., Cohen, C. F., and Joyner, S. C. (1968). Ecdysones and analogs - effects on development and reproduction of insects. Science 161, 1158-1160. doi: 10.1126/science.161.3846. 1158

Rybak, A., Fuchs, H., Smirnova, L., Brandt, C., Pohl, E. E., Nitsch, R., et al. (2008). A feedback loop comprising lin-28 and let-7 controls pre-let-7 maturation during neural stem-cell commitment. Nat. Cell Biol. 10, 987-993. doi: 10.1038/ ncb1759

Silver, D. L., and Montell, D. J. (2001). Paracrine signaling through the JAK/STAT pathway activates invasive behavior of ovarian epithelial cells in Drosophila. Cell 107, 831-841. doi: 10.1016/S0092-8674(01)00607-9

Sokol, N. S., Xu, P., Jan, Y. N., and Ambros, V. (2008). Drosophila let-7 microRNA is required for remodeling of the neuromusculature during metamorphosis. Genes Dev. 22, 1591-1596. doi: 10.1101/gad.1671708 
Song, Y., Ori-Mckenney, K. M., Zheng, Y., Han, C., Jan, L. Y., and Jan, Y. N. (2012). Regeneration of Drosophila sensory neuron axons and dendrites is regulated by the Akt pathway involving Pten and microRNA bantam. Genes Dev. 26, 1612-1625. doi: $10.1101 /$ gad.193243.112

Starz-Gaiano, M., Melani, M., Wang, X., Meinhardt, H., and Montell, D. J. (2008). Feedback inhibition of Jak/STAT signaling by apontic is required to limit an invasive cell population. Dev. Cell 14, 726-738. doi: 10.1016/j.devcel.2008. 03.005

Takeuchi, O., and Akira, S. (2010). Pattern recognition receptors and inflammation. Cell 140, 805-820. doi: 10.1016/j.cell.2010.01.022

Thompson, B. J., and Cohen, S. M. (2006). The Hippo pathway regulates the bantam microRNA to control cell proliferation and apoptosis in Drosophila. Cell 126, 767-774. doi: 10.1016/j.cell.2006.07.013

Wu, Y. C., Chen, C. H., Mercer, A., and Sokol, N. S. (2012). Let-7-complex microRNAs regulate the temporal identity of Drosophila mushroom body neurons via chinmo. Dev. Cell 23, 202-209. doi: 10.1016/j.devcel.2012. 05.013

Yoon, W. H., Meinhardt, H., and Montell, D. J. (2011). miRNA-mediated feedback inhibition of JAK/STAT morphogen signalling establishes a cell fate threshold. Nat. Cell Biol. 13, 1062-1069. doi: 10.1038/ncb2316

Zhu, S., Chiang, A. S., and Lee, T. (2003). Development of the Drosophila mushroom bodies: elaboration, remodeling and spatial organization of dendrites in the calyx. Development 130, 2603-2610. doi: 10.1242/dev.00466
Zhu, S., Lin, S., Kao, C. F., Awasaki, T., Chiang, A. S., and Lee, T. (2006). Gradients of the Drosophila Chinmo BTB-zinc finger protein govern neuronal temporal identity. Cell 127, 409-422. doi: 10.1016/j.cell.2006.08.045

Zou, Y., Chiu, H., Zinovyeva, A., Ambros, V., Chuang, C. F., and Chang, C. (2013). Developmental decline in neuronal regeneration by the progressive change of two intrinsic timers. Science 340, 372-376. doi: 10.1126/science.1231321

Conflict of Interest Statement: The authors declare that the research was conducted in the absence of any commercial or financial relationships that could be construed as a potential conflict of interest.

Received: 18 September 2013; paper pending published: 17 October 2013; accepted: 04 December 2013; published online: 24 December 2013.

Citation: Hartl M and Grunwald Kadow IC (2013) New roles for "old" microRNAs in nervous system function and disease. Front. Mol. Neurosci. 6:51. doi: 10.3389/fnmol. 2013.00051

This article was submitted to the journal Frontiers in Molecular Neuroscience.

Copyright $(2013$ Hartl and Grunwald Kadow. This is an open-access article distributed under the terms of the Creative Commons Attribution License (CC BY). The use, distribution or reproduction in other forums is permitted, provided the original author(s) or licensor are credited and that the original publication in this journal is cited, in accordance with accepted academic practice. No use, distribution or reproduction is permitted which does not comply with these terms. 